PROCEEDINGS OF THE

AMERICAN MATHEMATICAL SOCIETY

Volume 140, Number 5, May 2012, Pages 1643-1658

S 0002-9939(2011)11006-2

Article electronically published on August 22, 2011

\title{
GEOMETRIC RELATIONS BETWEEN SPACES OF NUCLEAR OPERATORS AND SPACES OF COMPACT OPERATORS
}

\author{
ELÓI MEDINA GALEGO AND RONALD PATERNINA SALGUEDO
}

(Communicated by Thomas Schlumprecht)

\begin{abstract}
We extend and provide a vector-valued version of some results of C. Samuel about the geometric relations between the spaces of nuclear operators $\mathcal{N}(E, F)$ and spaces of compact operators $\mathcal{K}(E, F)$, where $E$ and $F$ are Banach spaces $C(K)$ of all continuous functions defined on the countable compact metric spaces $K$ equipped with the supremum norm.

First we continue Samuel's work by proving that $\mathcal{N}\left(C\left(K_{1}\right), C\left(K_{2}\right)\right)$ contains no subspace isomorphic to $\mathcal{K}\left(C\left(K_{3}\right), C\left(K_{4}\right)\right)$ whenever $K_{1}, K_{2}, K_{3}$ and $K_{4}$ are arbitrary infinite countable compact metric spaces.

Then we show that it is relatively consistent with ZFC that the above result and the main results of Samuel can be extended to $C\left(K_{1}, X\right), C\left(K_{2}, Y\right)$, $C\left(K_{3}, X\right)$ and $C\left(K_{4}, Y\right)$ spaces, where $K_{1}, K_{2}, K_{3}$ and $K_{4}$ are arbitrary infinite totally ordered compact spaces; $X$ comprises certain Banach spaces such that $X^{*}$ are isomorphic to subspaces of $l_{1}$; and $Y$ comprises arbitrary subspaces of $l_{p}$, with $1<p<\infty$.

Our results cover the cases of some non-classical Banach spaces $X$ constructed by Alspach, by Alspach and Benyamini, by Benyamini and Lindenstrauss, by Bourgain and Delbaen and also by Argyros and Haydon.
\end{abstract}

\section{INTRODUCTION}

We follow the standard notation and terminology for set theory and Banach space theory that can be found in [24] and 25] respectively. Let $X$ and Y be Banach spaces and let $K$ be a compact Hausdorff space. $\mathcal{N}(X, Y)$ represents the space of nuclear operators from $X$ to $Y$ and $\mathcal{K}(X, Y)$ denotes the space of compact operators from $X$ to $Y$. By $C(K, X)$ we denote the Banach space of all continuous $X$-valued functions defined on $K$ and equipped with the supremum norm. This space will be denoted by $C(K)$ in the case $X=\mathbb{R}$. By $[0, \alpha]$ we denote the interval of ordinals endowed with the order topology. We write $X \sim Y$ when $X$ and $Y$ are isomorphic and $X \hookrightarrow Y$ when $Y$ contains a subspace isomorphic to $X$. When $Y$ contains a complemented subspace isomorphic to $X$, we also write $X \stackrel{c}{\hookrightarrow} Y$.

A very recent paper of C. Samuel [36] on spaces of operators on $C(K)$ spaces, where $K$ is a countable metric space, is the guideline for our work. Indeed, in [36, Theorem 2.4] it was proved that $\mathcal{K}(X, Y)$ contains no subspace isomorphic to $\mathcal{N}(X, Y)$, whenever $X$ and $Y$ are $C(K)$ spaces, with $K$ infinite countable metric

Received by the editors December 15, 2010 and, in revised form, January 12, 2011.

2010 Mathematics Subject Classification. Primary 46B03, 46B25; Secondary 47B10.

Key words and phrases. Isomorphic classifications of spaces of compact operators and spaces of nuclear operators.

(C)2011 American Mathematical Society Reverts to public domain 28 years from publication 
spaces. In other words, for all infinite countable metric compact spaces $K_{1}, K_{2}$, $K_{3}$ and $K_{4}$, we have

$$
\mathcal{N}\left(C\left(K_{1}\right), C\left(K_{2}\right)\right) \leftrightarrow \mathcal{K}\left(C\left(K_{3}\right), C\left(K_{4}\right)\right) .
$$

First of all, we prove that the following statement also holds:

$$
\mathcal{K}\left(C\left(K_{1}\right), C\left(K_{2}\right)\right) \leftrightarrow \mathcal{N}\left(C\left(K_{3}\right), C\left(K_{4}\right)\right) .
$$

Nevertheless, the other two main results of 36 show that the geometry of the spaces of nuclear operators and the geometry of the spaces of compact operators involved in (1) have some similarities. Namely, the following cancellation laws hold (see [36, Theorem 3.3] and [36, Theorem 4.2]):

$$
\begin{aligned}
\mathcal{N}\left(C\left(K_{1}\right), C\left(K_{1}\right)\right) & \sim \mathcal{N}\left(C\left(K_{2}\right), C\left(K_{2}\right)\right) \Longleftrightarrow C\left(K_{1}\right) \sim C\left(K_{2}\right), \\
\mathcal{K}\left(C\left(K_{1}\right), C\left(K_{1}\right)\right) & \sim \mathcal{K}\left(C\left(K_{2}\right), C\left(K_{2}\right)\right) \Longleftrightarrow C\left(K_{1}\right) \sim C\left(K_{2}\right) .
\end{aligned}
$$

Thus, the principal purpose of the present paper is to extend the above described Samuel theorems and (2) to $C\left(K_{1}, X\right), C\left(K_{2}, Y\right), C\left(K_{3}, X\right)$ and $C\left(K_{4}, Y\right)$ spaces, where $K_{1}, K_{2}, K_{3}$ and $K_{4}$ are totally ordered compact spaces, $X$ comprises certain Banach spaces such that $X^{*}$ are isomorphic to subspaces of $l_{1}$ and $Y$ comprises arbitrary subspaces of $l_{p}$ with $1<p<\infty$. Recall that a classical theorem of Mazurkiewicz and Sierpiński 32 states that every infinite countable compact metric space $K$ is homeomorphic to an interval of ordinals [0, $\alpha$, with $\alpha<\omega_{1}$.

Next we state our main results. Henceforth following [5], when $K$ is the interval of ordinals $[0, \alpha]$ and $X$ is a Banach space, the space $C(K, X)$ will be indicated by $X^{\alpha}$. We will write $X \hat{\otimes}_{\epsilon} Y$ for the injective tensor product of two given Banach spaces $X$ and $Y$, while the projective tensor product will be denoted by $X \hat{\otimes}_{\pi} Y$; see for instance [11. The cardinality of a set $\Gamma$ will be denote by $|\Gamma|$ and the cardinality of an ordinal $\alpha$ by $\bar{\alpha}$.

1.1. The generalization of (1). Since $l_{1}$ does not contain $l_{\infty}^{n}$ uniformly (see for instance [13, Remark 11.5.g] and [13, Theorem 14.1]), it follows that Theorem 1.1 contains the result (1) as a special case when $X=Y=\mathbb{R}$ and $\xi, \eta, \lambda$ and $\mu$ are infinite countable ordinals.

Theorem 1.1. Suppose that $X$ and $Y$ are Banach spaces such that $X^{*}$ or $Y$ has the approximation property and such that they satisfy

(a) $X^{*}$ is isomorphic to a subspace of $l_{1}$,

(b) $\left(\sum_{n=1}^{\infty} l_{\infty}^{n}\right)_{1} \nrightarrow l_{1} \hat{\otimes}_{\epsilon} Y$.

Then for all infinite ordinals $\lambda, \mu, \xi$ and $\eta$, we have

$$
\mathcal{N}\left(X^{\lambda}, Y^{\xi}\right) \hookrightarrow \mathcal{K}\left(X^{\mu}, Y^{\eta}\right)
$$

Remark 1.2. We will prove in Proposition 2.1 that

$$
\left(\sum_{n=1}^{\infty} l_{\infty}^{n}\right)_{1} \hookrightarrow l_{1} \hat{\otimes}_{\epsilon} l_{p}
$$

for every $1<p<\infty$. In particular, if $Y$ is a subspace of $l_{p}$, with $1<p<\infty$, then $Y$ satisfies the hypothesis of Theorem 1.1. 
1.2. The generalization of (2). Here it is convenient to introduce the following definition.

Definition 1.3. We say that a Banach space $X$ is a $\mathcal{N}_{0}$-space if for every Banach space $Y$ we have

$$
\mathcal{N}(X, Y) \hookrightarrow \mathcal{N}\left(c_{0}, Y\right) .
$$

Remark 1.4. Observe that if $X$ is an $\mathcal{N}_{0}$-space, then $X^{*}$ is isomorphic to a subspace of $l_{1}$. Further, finite dimensional spaces and Banach spaces which are isomorphic to $l_{1}$-preduals are, of course, examples of $\mathcal{N}_{0}$-spaces.

Theorem 1.5. Suppose that $X$ and $Y$ are Banach spaces such that $X^{*}$ or $Y$ has the approximation property. Assume that $X$ is an $\mathcal{N}_{0}$-space and $Y$ contains no subspace isomorphic to $l_{1}$. Then for all infinite ordinals $\lambda, \mu, \xi$ and $\eta$, we have

$$
\mathcal{K}\left(X^{\lambda}, Y^{\xi}\right) \nrightarrow \mathcal{N}\left(X^{\mu}, Y^{\eta}\right)
$$

1.3. The generalization of (3). In this case we need two definitions.

Definition 1.6. A Banach space $X$ is said to be an $\mathcal{N}_{1}$-space if for every set $\Gamma$ of cardinality $\aleph_{1}$ and for every Banach space $Y$ we have

$$
l_{1}(\Gamma) \stackrel{c}{\hookrightarrow} \mathcal{N}(X, Y) \Longrightarrow l_{1}(\Gamma) \stackrel{c}{\hookrightarrow} Y .
$$

Remark 1.7. It is clear that every finite dimensional space is an $\mathcal{N}_{1}$-space. Moreover, it follows directly from our Lemma 4.2 and [14, Theorem 1.10] that every Banach space which is isomorphic to an $l_{1}$-predual is an $\mathcal{N}_{1}$-space. In particular, every $C(K)$ space, with $K$ a countable metric space, is an $\mathcal{N}_{0^{-}}$and $\mathcal{N}_{1^{-}}$-space. Consequently, there are uncountably many non-isomorphic Banach spaces which are at the same time $\mathcal{N}_{0^{-}}$and $\mathcal{N}_{1}$-spaces [5]. Further, the 'non-classical' Banach spaces constructed in [1], [2], 3], 4] and [6] are also $\mathcal{N}_{0^{-}}$and $\mathcal{N}_{1}$-spaces, because every one of them is isomorphic to an $l_{1}$-predual.

Definition 1.8. We say that a Banach space $X$ is an $\mathcal{N}_{\infty}$-space if for every infinite ordinal $\alpha$ and for every Banach space $Y$ we have

$$
\mathbb{R}^{\alpha} \hookrightarrow \mathcal{N}(X, Y) \Longrightarrow \mathbb{R}^{\alpha} \hookrightarrow Y^{m} \text { for some } 1 \leq m<\omega .
$$

Remark 1.9. It is easy to see that if $X$ is an $\mathcal{N}_{0}$-space, then $X$ is an $\mathcal{N}_{\infty}$-space.

We recall that a Banach space $X$ is said to have the Mazur Property (in short, MP) if every element of $X^{* *}$ which is sequentially weak* continuous is weak* continuous and thus is an element of $X$. Such spaces were investigated in [15] and [27] and sometimes are also called $d$-complete [31] or $\mu \mathrm{B}$-spaces [39]. It is well known that every separable Banach spaces has the MP [32, Proposition 2.3].

Theorem 1.10. Suppose that $X$ is an $\mathcal{N}_{1}$-space and an $\mathcal{N}_{\infty}$-space and that $Y$ is a Banach space having the Mazur Property and containing no subspace isomorphic to $c_{0}$ and no complemented subspace isomorphic to $l_{1}(\Gamma)$ with $|\Gamma|=\aleph_{1}$. If $X^{*}$ or $Y$ has the approximation property, then for all infinite ordinals $\lambda, \mu, \xi$ and $\eta$ the following assertions are equivalent:

(a) $\mathcal{N}\left(X^{\lambda}, Y^{\xi}\right) \sim \mathcal{N}\left(X^{\mu}, Y^{\eta}\right)$.

(b) Either $\mathbb{R}^{\xi} \sim \mathbb{R}^{\eta}$ or $\mathbb{R}^{\xi} \sim \mathbb{R}^{\alpha p}$ and $\mathbb{R}^{\eta} \sim \mathbb{R}^{\alpha q}$ for some uncountable regular ordinal $\alpha$ and finite distinct ordinals $p \neq q$. Moreover, $\lambda$ and $\mu$ have the same cardinality. 
1.4. The generalization of (4). We begin by recalling that a cardinal number $\mathfrak{m}$ is a real-valued measurable cardinal if there is a non-trivial real-valued measure defined on all subsets of a set of cardinal $\mathfrak{m}$ for which points have measure zero [15, page 560]. We also need to introduce the following definition.

Definition 1.11. We say that the Banach space $X$ is a $\mathcal{K}_{0}$-space if for all infinite sets $I$ and $J$ we have

$$
\mathcal{K}\left(c_{0}(I), X\right) \hookrightarrow \mathcal{K}\left(c_{0}(J), X\right) \text { and } \mathcal{K}\left(c_{0}(J), X\right) \hookrightarrow \mathcal{K}\left(c_{0}(I), X\right) \Longrightarrow|I|=|J| .
$$

Remark 1.12. It is clear that every separable Banach space is a $\mathcal{K}_{0}$-space.

Theorem 1.13. Let $X$ be a Banach space such that $X^{*}$ is isomorphic to a subspace of $l_{1}$ and let $Y$ be a $\mathcal{K}_{0}$-space having the Mazur Property and containing no subspace isomorphic to $c_{0}$. Suppose that $X^{*}$ or $Y$ has the approximation property and that $\lambda, \mu, \xi$ and $\eta$ are infinite ordinals such that the cardinalities of $\lambda$ and $\mu$ are not real-valued measurable cardinals. Then following assertions are equivalent:

(a) $\mathcal{K}\left(X^{\lambda}, Y^{\xi}\right) \sim \mathcal{K}\left(X^{\mu}, Y^{\eta}\right)$.

(b) Either $\mathbb{R}^{\xi} \sim \mathbb{R}^{\eta}$ or $\mathbb{R}^{\xi} \sim \mathbb{R}^{\alpha p}$ and $\mathbb{R}^{\eta} \sim \mathbb{R}^{\alpha q}$ for some uncountable regular ordinal $\alpha$ and finite distinct ordinals $p \neq q$. Moreover, $\lambda$ and $\mu$ have the same cardinality.

Remark 1.14. It is well known that the existence of real-valued measurable cardinals cannot be proved in ZFC [26, pages 106 and 108]. On the other hand, it is relatively consistent with ZFC that real-valued measurable cardinals do not exist [21, Theorem 4.14, page 972]. So it is relatively consistent with ZFC that Theorem 1.13 provides a complete isomorphic classification of the spaces.

1.5. The main theorem. Now we are ready to state and prove the main theorem of this paper.

Theorem 1.15. Let $X$ be an $\mathcal{N}_{0}$-space with $X^{*}$ having the approximation property and let $Y$ be an arbitrary subspace of $l_{p}$, with $1<p<\infty$. Then for all infinite ordinals $\lambda, \mu, \xi$ and $\eta$, we have

(i) $\mathcal{N}\left(X^{\lambda}, Y^{\xi}\right) \nrightarrow \mathcal{K}\left(X^{\mu}, Y^{\eta}\right)$,

(ii) $\mathcal{K}\left(X^{\mu}, Y^{\eta}\right) \nrightarrow \mathcal{N}\left(X^{\lambda}, Y^{\xi}\right)$.

Furthermore, if $X$ is also an $\mathcal{N}_{1}$-space, then it is relatively consistent with ZFC that the following assertions are equivalent:

(iii) $\mathcal{N}\left(X^{\lambda}, Y^{\xi}\right) \sim \mathcal{N}\left(X^{\mu}, Y^{\eta}\right)$.

(iv) $\mathcal{K}\left(X^{\lambda}, Y^{\xi}\right) \sim \mathcal{K}\left(X^{\mu}, Y^{\eta}\right)$.

(v) Either $\mathbb{R}^{\xi} \sim \mathbb{R}^{\eta}$ or $\mathbb{R}^{\xi} \sim \mathbb{R}^{\alpha p}$ and $\mathbb{R}^{\eta} \sim \mathbb{R}^{\alpha q}$ for some uncountable regular ordinal $\alpha$ and finite distinct ordinals $p \neq q$. Moreover, $\lambda$ and $\mu$ have the same cardinality.

Proof. (i) By Remark $1.4 X^{*}$ is isomorphic to a subspace of $l_{1}$. So according to Remark 1.2 and Theorem 1.1 we are done.

(ii) Since $Y$ contains no subspace isomorphic to $l_{1}$, it suffices to apply Theorem 1.5.

To see that the assertions above are equivalent, notice that by Remark $1.9 \mathrm{X}$ is an $\mathcal{N}_{\infty}$-space. Moreover, every separable space has the Mazur Property; see for instance [27, Proposition 2.3]. Hence by Theorem 1.10, (iii) is equivalent to (v). 
On the other hand, by Remark $1.12 Y$ is a $\mathcal{K}_{\infty}$-space. Consequently Theorem 1.13 implies that (iv) is equivalent to (v) whenever $\lambda, \mu, \xi$ and $\eta$ are infinite ordinals such that the cardinalities of $\lambda$ and $\mu$ are not real-valued measurable cardinals. Therefore according to Remark 1.14, (iv) is equivalent to (v).

Remark 1.16. Even for $X=Y=\mathbb{R}$ the condition (v) of Theorem 1.15 cannot be improved. Indeed, suppose that $\alpha$ is an uncountable regular ordinal. Then it is easy to check that

$$
\mathcal{N}\left(\mathbb{R}^{\alpha}, \mathbb{R}^{\alpha}\right) \sim \mathcal{N}\left(\mathbb{R}^{\alpha 2}, \mathbb{R}^{\alpha 2}\right) \text { and } \mathcal{K}\left(\mathbb{R}^{\alpha}, \mathbb{R}^{\alpha}\right) \sim \mathcal{K}\left(\mathbb{R}^{\alpha 2}, \mathbb{R}^{\alpha 2}\right) .
$$

However by [28, Theorem 2] we know that $\mathbb{R}^{\alpha} \nsim \mathbb{R}^{\alpha 2}$.

Remark 1.17. It follows from Theorem 1.15 that Samuel's above-mentioned theorems hold also for a large family of non-metrizable compact spaces $K_{i}, 1 \leq i \leq 4$. Indeed, take $X=Y=\mathbb{R}, \beta$ a singular ordinal and $\lambda, \mu, \xi$ and $\eta$ ordinals of the same cardinality as $\beta$. So, condition (v) of Theorem 1.15 is reduced to $\mathbb{R}^{\xi} \sim \mathbb{R}^{\eta}$.

From now on, our task is to prove Theorem 1.1 (Section 2), Theorem 1.5 (Section 3), Theorem 1.10 (Section 4) and Theorem 1.13 (Section 5).

\section{On embedding of $\mathcal{N}\left(X^{\lambda}, Y^{\xi}\right)$ into $\mathcal{K}\left(X^{\mu}, Y^{\eta}\right)$}

The main aim of this section is to prove the generalization of (1). That is:

Proof of Theorem 1.1. Assume to the contrary that there exist some infinite ordinals $\lambda, \mu, \xi$ and $\eta$ such that

$$
\mathcal{N}\left(X^{\lambda}, Y^{\xi}\right) \hookrightarrow \mathcal{K}\left(X^{\mu}, Y^{\eta}\right)
$$

Notice that if $X^{*}$ has the approximation property, then by [11, Proposition 5.8], $l_{1}\left([0, \alpha], X^{*}\right)$ also has this property. Further, if $Y$ has the approximation property, then since $\mathbb{R}^{\alpha}$ has the same property for every ordinal $\alpha$ [12, page 245], it follows that $Y^{\alpha}$ has the approximation property for every ordinal $\alpha$ 23. Consequently by [11, Corollary, page 65] and [11, Proposition 5.3], we obtain respectively

$$
\mathcal{N}\left(X^{\lambda}, Y^{\xi}\right) \sim l_{1}\left([0, \lambda], X^{*}\right) \hat{\otimes}_{\pi} Y^{\xi} \text { and } \mathcal{K}\left(X^{\mu}, Y^{\eta}\right) \sim l_{1}\left([0, \mu], X^{*}\right) \hat{\otimes}_{\epsilon} Y^{\eta}
$$

Since $\lambda$ and $\xi$ are infinite and $l_{1}[0, \lambda]$ is a complemented subspace of $l_{1}\left([0, \lambda], X^{*}\right)$ by [14, page 41] and [38, section 20.3.7.B],

$$
l_{1}[0, \lambda] \hat{\otimes}_{\pi} Y^{\xi} \stackrel{c}{\hookrightarrow} l_{1}\left([0, \lambda], X^{*}\right) \hat{\otimes}_{\pi} Y^{\xi} .
$$

Furthermore, by [14, Theorem 1.10],

$$
l_{1}\left([0, \lambda], Y^{\xi}\right) \sim l_{1}[0, \lambda] \hat{\otimes}_{\pi} Y^{\xi} .
$$

But, of course, $\left(\sum_{n=1}^{\infty} l_{\infty}^{n}\right)_{1} \hookrightarrow l_{1}\left([0, \lambda], Y^{\xi}\right)$. So according to (7) and (8)

$$
\left(\sum_{n=1}^{\infty} l_{\infty}^{n}\right)_{1} \hookrightarrow l_{1}\left([0, \lambda], X^{*}\right) \hat{\otimes}_{\pi} Y^{\xi}
$$

On the other hand, it follows by (a) of Theorem 1.1 that

$$
l_{1}\left([0, \mu], X^{*}\right) \hookrightarrow l_{1}\left([0, \mu], l_{1}\right) \sim l_{1}[0, \mu] .
$$

Hence

$$
l_{1}\left([0, \mu], X^{*}\right) \hat{\otimes}_{\epsilon} Y^{\eta} \hookrightarrow l_{1}[0, \mu] \hat{\otimes}_{\epsilon} Y^{\eta}
$$


Moreover, by [38, Theorem 20.5.6] we see that

$$
l_{1}[0, \mu] \hat{\otimes}_{\epsilon} Y^{\eta} \sim l_{1}[0, \mu] \hat{\otimes}_{\epsilon}\left(Y \hat{\otimes}_{\epsilon} \mathbb{R}^{\eta}\right) \sim \mathbb{R}^{\eta} \hat{\otimes}_{\epsilon}\left(l_{1}[0, \mu] \hat{\otimes}_{\epsilon} Y\right) \sim\left(l_{1}[0, \mu] \hat{\otimes}_{\epsilon} Y\right)^{\eta} .
$$

So

$$
l_{1}\left([0, \mu], X^{*}\right) \hat{\otimes}_{\epsilon} Y^{\eta} \hookrightarrow\left(l_{1}[0, \mu] \hat{\otimes}_{\epsilon} Y\right)^{\eta} .
$$

Therefore by (5), (6), (9) and (10) we see that

$$
\left(\sum_{n=1}^{\infty} l_{\infty}^{n}\right)_{1} \hookrightarrow\left(l_{1}[0, \mu] \hat{\otimes}_{\epsilon} Y\right)^{\eta} .
$$

However, it is not difficult to check that $\left(\sum_{n=1}^{\infty} l_{\infty}^{n}\right)_{1}$ contains no subspace isomorphic to $c_{0}$ and that $l_{1}[0, \mu] \hat{\otimes}_{\epsilon} Y$ is isomorphic to its own square. Consequently by (11) and [18, Theorem 2.3] we deduce that

$$
\left(\sum_{n=1}^{\infty} l_{\infty}^{n}\right)_{1} \hookrightarrow l_{1}[0, \mu] \hat{\otimes}_{\epsilon} Y .
$$

Thus by the separability of $\left(\sum_{n=1}^{\infty} l_{\infty}^{n}\right)_{1}$, we conclude

$$
\left(\sum_{n=1}^{\infty} l_{\infty}^{n}\right)_{1} \hookrightarrow l_{1} \hat{\otimes}_{\epsilon} Y .
$$

This contradicts (b) and the proof is complete.

Observe that [7, Proposition 1] states that $Y=c_{0}$ satisfies the hypothesis of Theorem 1.1. Next, inspired by this proposition, we will show that the same happens with $Y=l_{p}$ with $1<p<\infty$. In this case, we will need some results of [35].

Proposition 2.1. Suppose that $1<p<\infty$. Then $\left(\sum_{n=1}^{\infty} l_{\infty}^{n}\right)_{1} \nrightarrow l_{1} \hat{\otimes}_{\epsilon} l_{p}$.

Proof. Following [35] we consider the Banach space

$$
s l_{p}\left(l_{1}\right)=\left\{u=\left(x_{n}\right)_{n \in \mathbb{N}} \text { with } x_{n} \in l_{1}, \quad \forall n \in \mathbb{N}: \sum_{n=1}^{\infty}\left|x^{*}\left(x_{n}\right)\right|^{p}<\infty, \quad \forall x^{*} \in l_{1}^{*}\right\},
$$

with $\|u\|=\sup \left\{\left(\sum_{n=1}^{\infty}\left|x^{*}\left(x_{n}\right)\right|^{p}\right)^{1 / p}: x^{*} \in l_{1}^{*}\right.$ and $\left.\left\|x^{*}\right\| \leq 1\right\}$. For every $m \in \mathbb{N}$, we denote by $P_{m}: s l_{p}\left(l_{1}\right) \rightarrow s l_{p}\left(l_{1}\right)$ the linear operator given by $P_{m}\left(\left(x_{n}\right)_{n \in \mathbb{N}}\right)=$ $\left(y_{n}\right)_{n \in \mathbb{N}}$, where $y_{n}=x_{n}$ for every $n \leq m$ and $y_{n}=0$ for every $n>m$. We also denote $F_{p}\left(l_{1}\right)=\left\{u \in s l_{p}\left(l_{1}\right): \lim _{m \rightarrow \infty} P_{m}(u)=u\right\}$. Then by [35, page 106], $F_{p}\left(l_{1}\right)$ is isometric to $l_{p} \hat{\otimes}_{\epsilon} l_{1}$.

We will assume by contradiction that $F_{p}\left(l_{1}\right)$ contains a subspace isomorphic to $\left(\sum_{n=1}^{\infty} l_{\infty}^{n}\right)_{1}$. Then there exists a linear operator $T:\left(\sum_{n=1}^{\infty} l_{\infty}^{n}\right)_{1} \rightarrow F_{p}\left(l_{1}\right)$ and $M \geq 1$ such that, for every $x \in\left(\sum_{n=1}^{\infty} l_{\infty}^{n}\right)_{1}$, we have

$$
1 / M\|x\| \leq\|T(x)\| \leq M\|x\| .
$$

Notice that for every $m \in \mathbb{N}, P_{m}\left(F_{p}\left(l_{1}\right)\right)$ is isomorphic to $\left(l_{1}\right)^{m}$ which in turn is isomorphic to $l_{1}$. Hence $P_{m}\left(F_{p}\left(l_{1}\right)\right)$ does not contain $l_{\infty}^{n}$ 's uniformly. Thus $P_{m} T$ cannot be an isomorphism onto its image. Therefore, for every $\epsilon>0$, there exists $z$ in the unit sphere of $\left(\sum_{n=1}^{\infty} l_{\infty}^{n}\right)_{1}$ such that $\left\|P_{m} T(z)\right\| \leq \epsilon$.

Thus, by using induction and a "gliding hump" argument, as in the proof of [7, Proposition 1], we can find a sequence $\left(z_{k}\right)_{k \in \mathbb{N}}$ in the unit sphere of $\left(\sum_{n=1}^{\infty} l_{\infty}^{n}\right)_{1}$ and an increasing sequence $\left(m_{k}\right)_{k \in \mathbb{N}}$ in $\mathbb{N}$ such that for every $k \in \mathbb{N}$,

(i) $\left\|P_{m_{k}} T\left(z_{k}\right)\right\|<1 / 2^{k}$, 
(ii) $\left\|\left(P_{m_{k+1}}-P_{m_{k}}\right) T\left(z_{k}\right)\right\|>1 / 2 M$,

(iii) $\left\|T\left(z_{k}\right)-P_{m_{k+1}} T\left(z_{k}\right)\right\|<1 / 2^{k+1}$.

For every $k \in \mathbb{N}$, denote $w_{k}=\left(P_{m_{k+1}}-P_{m_{k}}\right) T\left(z_{k}\right)$ and $u_{k}=T\left(z_{k}\right)-w_{k}$. According to Rosenthal's $l_{1}$-theorem [30, Theorem 2.e.5], by passing to a subsequence we may assume that $\left(z_{k}\right)_{k \in \mathbb{N}}$ is weakly Cauchy or is equivalent to the unit vector basis of $l_{1}$. In both cases we will get a contradiction.

In the first case, since $\left(\sum_{n=1}^{\infty} l_{\infty}^{n}\right)_{1}$ has Schur property, $\left(z_{k}\right)_{k \in \mathbb{N}}$ converges in norm to some $z$ in $\left(\sum_{n=1}^{\infty} l_{\infty}^{n}\right)_{1}$. On the other hand, by (i) and (iii) we see that $\left(u_{k}\right)_{k \in \mathbb{N}}$ is a null sequence in $F_{p}\left(l_{1}\right)$. Moreover, according to (ii), $\left(w_{k}\right)_{k \in \mathbb{N}}$ is a semi-normalized disjointly supported sequence in $F_{p}\left(l_{1}\right)$. Therefore it is a null sequence in $F_{p}\left(l_{1}\right)$. Hence $T z=0$, which is of course absurd, since (12) holds.

In the second case, by (i) and (iii), for every $k \in \mathbb{N}$, we have $\left\|T\left(z_{k}\right)-w_{k}\right\|<1 / 2^{k}$. Thus by the classical perturbation result [30, Proposition 1.a.9], by passing to a further subsequence we may assume that $v_{k}=w_{k} /\left\|w_{k}\right\|$ is also equivalent to the unit vector basis of $l_{1}$.

Next, we need to recall some more definitions of 35 . For every $m \in \mathbb{N}$, define $Q_{m}: l_{1} \rightarrow l_{1}$ by $Q_{m}\left(\left(a_{n}\right)_{n \in \mathbb{N}}\right)=\left(b_{n}\right)_{n \in \mathbb{N}}$, where $b_{n}=a_{n}$ for every $n \leq m$ and $b_{n}=0$ for every $n>m$.

Define also $\widetilde{Q}_{m}: F_{p}\left(l_{1}\right) \rightarrow F_{p}\left(l_{1}\right)$ by $\widetilde{Q}_{m}\left(\left(x_{n}\right)_{n \in \mathbb{N}}\right)=\left(Q_{m}\left(x_{n}\right)\right)_{n \in \mathbb{N}}$. Then, for every $m \in \mathbb{N},\left\|\tilde{Q}_{m}\right\|=1$ and by [35, Lemma 1] we know that

$$
\widetilde{Q}_{m}\left(F_{p}\left(l_{1}\right)\right) \sim l_{p}, \quad \forall m \in \mathbb{N}
$$

Furthermore, according to [35, Lemma 2] we have

$$
\lim _{m \rightarrow \infty} \widetilde{Q}_{m}(x)=x, \quad \forall x \in F_{p}\left(l_{1}\right) .
$$

Since $p>1$, it follows by (13) that $\widetilde{Q}_{1}$ restricted to the closed linear span of $\left\{v_{i}\right.$ : $i \in \mathbb{N}\}$ in $F_{p}\left(l_{1}\right)$ is not an isomorphism onto its image. So, there exist $k_{1} \in \mathbb{N}$ and $v_{1}^{\prime}$ in the unit sphere of the closed linear span of $\left\{v_{i}: 1 \leq i \leq k_{1}\right\}$ in $F_{p}\left(l_{1}\right)$ such that $\left\|\tilde{Q}_{1}\left(v_{1}^{\prime}\right)\right\|<1 / 2$. By $(14)$, there exists $n_{2}>1=n_{1}$ such that $\left\|v_{1}^{\prime}-\widetilde{Q}_{n_{2}}\left(v_{1}^{\prime}\right)\right\|<\delta / 2$. Again $\widetilde{Q}_{n_{2}}$ restricted to the closed linear span of $\left\{v_{i}: i \geq k_{1}+1\right\}$ in $F_{p}\left(l_{1}\right)$ is not an isomorphism onto its image. Thus there exist $k_{2} \in \mathbb{N}$ and $v_{2}^{\prime}$ in the unit sphere of the closed linear span of $\left\{v_{i}: k_{1}+1 \leq i \leq k_{2}\right\}$ in $F_{p}\left(l_{1}\right)$ such that $\left\|\widetilde{Q}_{1}\left(v_{2}^{\prime}\right)\right\|<1 / 2^{2}$. By induction, we obtain a block basic sequence $\left(v_{k}^{\prime}\right)_{k \in \mathbb{N}}$ of $\left(v_{k}\right)_{k \in \mathbb{N}}$ such that for every $k \in \mathbb{N}$,

$$
\begin{aligned}
& \text { (iv) }\left\|\tilde{Q}_{n_{k}}\left(v_{k}^{\prime}\right)\right\|<1 / 2^{k}, \\
& \text { (v) }\left\|\left(\tilde{Q}_{n_{k+1}}-\tilde{Q}_{n_{k}}\right)\left(v_{k}^{\prime}\right)-v_{k}^{\prime}\right\|<1 / 2^{k} .
\end{aligned}
$$

Put

$$
w_{k}^{\prime}=\frac{\left(\tilde{Q}_{n_{k+1}}-\tilde{Q}_{n_{k}}\right)\left(v_{k}^{\prime}\right)}{\left\|\left(\tilde{Q}_{n_{k+1}}-\widetilde{Q}_{n_{k}}\right)\left(v_{k}^{\prime}\right)\right\|} .
$$

Again by the classical perturbation result [30, Proposition 1.a.9], by passing to a subsequence we may assume that $\left(w_{k}^{\prime}\right)_{k \in \mathbb{N}}$ is equivalent to the unit vector basis of $l_{1}$. Moreover, observe that we have constructed two strictly increasing sequences $\left(m_{k}\right)_{k \in \mathbb{N}}$ and $\left(n_{k}\right)_{k \in \mathbb{N}}$ in $\mathbb{N}$ with $n_{1}=1$ and $m_{1}=1$ and a normalized sequence $\left(w_{k}^{\prime}\right)_{k \in \mathbb{N}}$ 
in $F_{p}\left(l_{1}\right)$ such that for every $k \in \mathbb{N}, w_{k}^{\prime}=\left(P_{n_{k}}-P_{n_{k-1}}\right)\left(w_{k}^{\prime}\right)=\left(\widetilde{Q}_{m_{k}}-\widetilde{Q}_{m_{k-1}}\right)\left(w_{k}^{\prime}\right)$. Hence according to the proof of the main theorem of $35,\left(w_{k}^{\prime}\right)_{k \in \mathbb{N}}$ is equivalent to the unit vector basis of $l_{p}$, a contradiction because $l_{1}$ is not isomorphic to $l_{p}$, with $1<p<\infty$.

\section{On EMBedDing of $\mathcal{K}\left(X^{\lambda}, Y^{\xi}\right)$ INTO $\mathcal{N}\left(X^{\mu}, Y^{\eta}\right)$}

The main goal of this section is the generalization of (2). In order to do this we need to state Proposition 3.1. It is an adaptation of [8, Lemma 1] and [8, Proposition 3]. For the sake of completeness, we give the proof of this proposition.

Proposition 3.1. Let $X$ be a Banach space such that $c_{0}\left(l_{1}\right) \hookrightarrow l_{1}(\mathbb{N}, X)$. Then there exists $1 \leq m<\omega$ such that $c_{0}\left(l_{1}\right) \hookrightarrow X^{m}$.

Proof. Let $T: c_{0}\left(l_{1}\right) \rightarrow l_{1}(\mathbb{N}, X)$ be an isomorphism onto its image and let $M \geq 1$ be such that for all $x \in c_{0}\left(l_{1}\right)$ we have $1 / M\|x\| \leq\|T(x)\| \leq M\|x\|$. Suppose that $c_{0}\left(l_{1}\right) \leftrightarrow X^{m}$, for every $1 \leq m<\omega$. For every $m \in \mathbb{N}$, denote by $P_{m}: c_{0}\left(l_{1}\right) \rightarrow$ $c_{0}\left(l_{1}\right)$ the linear operator given by $P_{m}\left(\left(x_{n}\right)_{n \in \mathbb{N}}\right)=\left(y_{n}\right)_{n \in \mathbb{N}}$, where $y_{n}=x_{n}$ for every $n \leq m$ and $y_{n}=0$ for every $n>m$. Denote also by $Q_{m}: l_{1}(\mathbb{N}, X) \rightarrow l_{1}(\mathbb{N}, X)$ the linear operator given by $Q_{m}\left(\left(x_{n}\right)_{n \in \mathbb{N}}\right)=\left(y_{n}\right)_{n \in \mathbb{N}}$, where $y_{n}=x_{n}$ for every $n \leq m$ and $y_{n}=0$ for every $n>m$. Let $I$ be the identity operator in $c_{0}\left(l_{1}\right)$. Observe that $\left(I-P_{n}\right)\left(c_{0}\left(l_{1}\right)\right)$ is isomorphic to $c_{0}\left(l_{1}\right)$ and $Q_{m}\left(l_{1}(\mathbb{N}, X)\right)$ is isomorphic to $X^{m}$, for every $m \in \mathbb{N}$. Therefore, $Q_{m} T_{\mid\left(I-P_{n}\right)\left(c_{0}\left(l_{1}\right)\right)}$ is not an isomorphism onto its image. Pick $m \in \mathbb{N}$. Thus, for each $\epsilon>0$ there exists $y$ in the unit sphere of $\left(I-P_{n}\right)\left(c_{0}\left(l_{1}\right)\right)$ such that $\left\|Q_{m} T(y)\right\|<\epsilon$. Since $y=\lim _{j} P_{j}(y)=\lim _{j}\left(P_{j}-P_{n}\right)(y)$, there exists $n^{\prime}>n$ such that $z=\left(P_{n^{\prime}}-P_{n}\right)(y), 3 / 4<\|z\| \leq 1$ and $\left\|Q_{m} T(z)\right\|<\epsilon$.

Therefore, by a "gliding hump" argument, as in the proof of [8, Proposition 3], we can find by induction a sequence $\left(z_{j}\right)_{j \in \mathbb{N}}$ in the unit sphere of $c_{0}\left(l_{1}\right)$ and two increasing sequence $\left(k_{j}\right)_{j \in \mathbb{N}},\left(m_{j}\right)_{j \in \mathbb{N}}$ in $\mathbb{N}$ such that for every $j \in \mathbb{N}$ :

(i) $z_{j}=\left(P_{k_{j+1}}-P_{k_{j}}\right) z_{j}, 3 / 4<\left\|z_{j}\right\| \leq 1$,

(ii) $\left\|Q_{m_{j+1}} T\left(z_{j}\right)\right\|<1 / 2^{j}$,

(iii) $\left\|\left(Q_{m_{j+1}}-Q_{m_{j}}\right) T\left(z_{j}\right)\right\|>1 / 2 M$,

(iv) $\left\|T\left(z_{j}\right)-Q_{m_{j+1}} T\left(z_{j}\right)\right\|<1 / 2^{j+1}$.

For every $j \in \mathbb{N}$, denote $w_{j}=\left(Q_{m_{j+1}}-Q_{m_{j}}\right) T\left(z_{j}\right)$ and $u_{j}=T\left(z_{j}\right)-w_{j}$. Notice that by $(\mathrm{i}),\left(z_{j}\right)_{j \in \mathbb{N}}$ is a normalized disjointly supported sequence in $c_{0}\left(l_{1}\right)$. Hence it is equivalent to the unit vector basis of $c_{0}$. On the other hand, according to (i) and (iii), $\left(w_{j}\right)_{j \in \mathbb{N}}$ is a normalized disjointly supported sequence in $l_{1}(\mathbb{N}, X)$. Consequently, it is equivalent to the unit vector basis of $l_{1}$. Moreover, by (ii) and (iv), $\left(u_{j}\right)_{j \in \mathbb{N}}$ is a null sequence in $l_{1}(\mathbb{N}, X)$.

Since $z_{j}=w_{j}+u_{j}$, by (iii) and the classical perturbation result 30, Proposition 1.a.9], by passing to a subsequence we conclude that $\left(T\left(z_{j}\right)\right)_{j \in \mathbb{N}}$ is equivalent to $\left(w_{j}\right)_{j \in \mathbb{N}}$, which contradicts the well-known fact that $c_{0}$ is not isomorphic to $l_{1}$. This completes the proof of the proposition.

Proof of Theorem 1.5. We assume that there exist some infinite ordinals $\lambda, \mu, \xi$ and $\eta$ such that

$$
\mathcal{K}\left(X^{\lambda}, Y^{\xi}\right) \hookrightarrow \mathcal{N}\left(X^{\mu}, Y^{\eta}\right)
$$

and argue to a contradiction. 
First, as in the beginning of the proof of Theorem 1.1, notice that

$$
\mathcal{K}\left(X^{\lambda}, Y^{\xi}\right) \sim l_{1}\left([0, \lambda], X^{*}\right) \hat{\otimes}_{\epsilon} Y^{\xi} \text { and } \mathcal{N}\left(X^{\mu}, Y^{\eta}\right) \sim l_{1}\left([0, \mu], X^{*}\right) \hat{\otimes}_{\pi} Y^{\eta}
$$

Moreover,

$$
c_{0}\left(l_{1}\right) \sim l_{1} \hat{\otimes}_{\epsilon} c_{0} \hookrightarrow l_{1}[0, \lambda] \hat{\otimes}_{\epsilon} Y^{\xi} \hookrightarrow l_{1}\left([0, \lambda], X^{*}\right) \hat{\otimes}_{\epsilon} Y^{\xi} .
$$

On the other hand, by [38, page 358] and [14, Theorem 1.10], we have

$$
l_{1}\left([0, \mu], X^{*}\right) \hat{\otimes}_{\pi} Y^{\eta} \sim l_{1}[0, \mu] \hat{\otimes}_{\pi}\left(X^{*} \hat{\otimes}_{\pi} Y^{\eta}\right) \sim l_{1}\left([0, \mu], X^{*} \hat{\otimes}_{\pi} Y^{\eta}\right) .
$$

Since $X$ is an $\mathcal{N}_{0}$-space, it follows that $X^{*} \hat{\otimes}_{\pi} Y^{\eta} \hookrightarrow l_{1}\left(\mathbb{N}, Y^{\eta}\right)$. Hence

$$
l_{1}\left([0, \mu], X^{*} \hat{\otimes}_{\pi} Y^{\eta}\right) \hookrightarrow l_{1}\left([0, \mu], l_{1}\left(\mathbb{N}, Y^{\eta}\right)\right) \sim l_{1}\left([0, \mu], Y^{\eta}\right) .
$$

Consequently by (15), (16), (17), (18) and (19) we have $c_{0}\left(l_{1}\right) \hookrightarrow l_{1}\left([0, \mu], Y^{\eta}\right)$. Therefore by the separability of $c_{0}\left(l_{1}\right)$ we deduce $c_{0}\left(l_{1}\right) \hookrightarrow l_{1}\left(\mathbb{N}, Y^{\eta}\right)$. Thus, by Proposition 3.1 there exists $1 \leq m<\omega$ such that $l_{1} \hookrightarrow c_{0}\left(l_{1}\right) \hookrightarrow\left(Y^{\eta}\right)^{m} \sim Y^{\eta m}$. Then, by [18, Theorem 3.2], there exists $1 \leq n<\omega$ such that $l_{1} \hookrightarrow Y^{n}$, and [35, Theorem 1] implies that $l_{1} \hookrightarrow Y$; this gives us the required contradiction.

\section{On isomorphic Classifications of $\mathcal{N}\left(X^{\lambda}, Y^{\xi}\right)$ SPACES}

The aim of this fourth section is to prove the generalization of (3). In order to prove it, we state some previous auxiliary results.

Lemma 4.1. Let $I$ and $J$ be sets with $|I|>|J|$ and $I$ uncountable. Suppose that $X$ is a Banach space such that $l_{\infty}(J, X)$ contains a subspace isomorphic to $l_{\infty}(I)$. Then $X$ contains a subspace isomorphic to $l_{\infty}(\Gamma)$, with $|\Gamma|=\aleph_{1}$.

Proof. First we assume that $|I|$ is a regular cardinal. By hypothesis there exists an isomorphism $T$ from $l_{\infty}(I)$ onto a subspace of $l_{\infty}(J, X)$. Let $M>0$ be such that $M \leq\|T(x)\|, \forall x \in l_{\infty}(I),\|x\|=1$. Denote by $\left(e_{i}\right)_{i \in I}$ the unit vectors of $l_{\infty}(I)$; that is, $e_{i}(j)=1$ if $i=j, e_{i}(j)=0$ if $i \neq j$, for all $i, j \in I$. For fixed $j \in J$, we define $I_{j}=\left\{i \in I: M / 2 \leq\left\|T\left(e_{i}\right)(j)\right\|\right\}$. Therefore $I=\bigcup_{j \in J} I_{j}$. Hence there is a $j \in J$ satisfying $\left|I_{j}\right|=|I|$. We identify $l_{\infty}\left(I_{j}\right)$ with the subspace of $l_{\infty}(I)$ consisting of those elements $f$ such that $f(\gamma)=0$ for every $\gamma \notin I_{j}$. Let $P_{j}: l_{\infty}(J, X) \rightarrow X$ be the natural projection; that is, $P_{j}(f)=f(j)$, for all $f \in l_{\infty}(J, X)$. Next, consider the linear operator $L=P_{j} T_{\mid l_{\infty}\left(I_{j}\right)}: l_{\infty}\left(I_{j}\right) \rightarrow X$. Then $\inf \left\{\left\|L\left(e_{i}\right)\right\|: i \in I_{j}\right\}>0$. So, according to Remark 1 after [33, Proposition 1.2], there exists $\Gamma \subset I_{j}$ with $|\Gamma|=$ $\left|I_{j}\right|$ such that $L_{\mid l_{\infty}(\Gamma)}$ is an isomorphism onto its image. So we are done.

Let us now suppose that $|I|$ is a singular cardinal. Then there exists a limit ordinal $\lambda$ such that $|I|=\aleph_{\lambda}$. Let $\gamma$ be an ordinal satisfying $|J|<\aleph_{\gamma+1}<\aleph_{\lambda}$. It is well known that $\aleph_{\gamma+1}$ is a regular cardinal. Let $\Lambda$ be a set of cardinality $\aleph_{\gamma+1}$. By hypothesis $l_{\infty}(\Lambda) \hookrightarrow l_{\infty}(I) \hookrightarrow l_{\infty}(J, X)$. Hence according to what we have just proved $X$ contains a subspace isomorphic to $l_{\infty}(\Gamma)$, with $|\Gamma|=\aleph_{1}$.

Lemma 4.2. Let $I$ and $J$ be sets with $|I|>|J|$ and $I$ uncountable. Suppose that $X$ is a Banach space such that $l_{1}(J, X)$ contains a complemented subspaces isomorphic to $l_{1}(I)$. Then $X$ contains a complemented subspace isomorphic to $l_{1}(\Gamma)$, with $|\Gamma|=\aleph_{1}$. 
Proof. Since $l_{1}(J, X)$ contains a complemented subspace isomorphic to $l_{1}(I)$, it follows that $l_{\infty}(I) \hookrightarrow l_{\infty}\left(J, X^{*}\right)$. By Lemma 4.1 there exists a set $\Gamma$ with $|\Gamma|=\aleph_{1}$ such that $l_{\infty}(\Gamma) \hookrightarrow X^{*}$. Hence by [34, Corollary 1.2], $X$ contains a complemented subspace isomorphic to $l_{1}(\Gamma)$.

Lemma 4.3. Suppose that $X$ is an $\mathcal{N}_{1}$-space and that $Y$ is a Banach space containing no complemented subspace isomorphic to $l_{1}(\Gamma)$, with $|\Gamma|=\aleph_{1}$. Assume that $X^{*}$ or $Y$ has the approximation property and

$$
l_{1}\left([0, \lambda], X^{*}\right) \hat{\otimes}_{\pi} Y^{\xi} \sim l_{1}\left([0, \mu], X^{*}\right) \hat{\otimes}_{\pi} Y^{\eta},
$$

for some infinite ordinals $\lambda, \mu, \xi$ and $\eta$. Then $\bar{\lambda}=\bar{\mu}$.

Proof. Suppose that $\bar{\lambda} \neq \bar{\mu}$. We can assume without loss of generality that $\bar{\lambda}<$ $\bar{\mu}$. Since $\mathbb{R}$ is a complemented subspace of $Y^{\eta}$, by [14, page 41] and [38, section 20.3.7.B], we see that

$$
l_{1}[0, \mu] \sim l_{1}[0, \mu] \hat{\otimes}_{\pi} \mathbb{R} \stackrel{c}{\hookrightarrow} l_{1}[0, \mu] \hat{\otimes}_{\pi} Y^{\eta} .
$$

Moreover, $l_{1}[0, \mu]$ is a complemented subspace of $l_{1}\left([0, \mu], X^{*}\right)$. Thus, again by $[14$, page 41] and [38, section 20.3.7.B], we have

$$
l_{1}[0, \mu] \hat{\otimes}_{\pi} Y^{\eta} \stackrel{c}{\hookrightarrow} l_{1}\left([0, \mu], X^{*}\right) \hat{\otimes}_{\pi} Y^{\eta} .
$$

On the other hand, by [14, Theorem 1.10], $l_{1}\left([0, \lambda], X^{*}\right) \sim l_{1}[0, \lambda] \hat{\otimes}_{\pi} X^{*}$. So by [38, section 20.3.7.B],

$$
l_{1}\left([0, \lambda], X^{*}\right) \hat{\otimes}_{\pi} Y^{\eta} \sim\left(l_{1}\left([0, \lambda] \hat{\otimes}_{\pi} X^{*}\right) \hat{\otimes}_{\pi} Y^{\eta} .\right.
$$

Now by [38, section 20.3.3] and again [14, Theorem 1.10] we deduce

$$
\left(l_{1}[0, \lambda] \hat{\otimes}_{\pi} X^{*}\right) \hat{\otimes}_{\pi} Y^{\xi} \sim l_{1}[0, \lambda] \hat{\otimes}_{\pi}\left(X^{*} \hat{\otimes}_{\pi} Y^{\xi}\right) \sim l_{1}\left([0, \lambda], X^{*} \hat{\otimes}_{\pi} Y^{\xi}\right) .
$$

According to our hypothesis, (20), (21), (22) and (23), $l_{1}[0, \mu] \stackrel{c}{\hookrightarrow} l_{1}\left([0, \lambda], X^{*} \hat{\otimes}_{\pi} Y^{\xi}\right)$. Therefore, by Lemma 4.2 , there exists a set $\Gamma$ with cardinality $\aleph_{1}$ such that $l_{1}(\Gamma) \stackrel{c}{\hookrightarrow}$ $X^{*} \hat{\otimes}_{\pi} Y^{\xi}$. But as we observed in the beginning of the proof of Theorem 1.1, if $Y$ has the approximation property, then $Y^{\eta}$ also has this property. So by our hypothesis and [1, Corollary of page 65], we infer that $l_{1}(\Gamma) \stackrel{c}{\hookrightarrow} \mathcal{N}\left(X, Y^{\xi}\right)$. Since $X$ is an $\mathcal{N}_{1}$-space, it follows that $l_{1}(\Gamma) \stackrel{c}{\hookrightarrow} Y^{\xi}$. But $c_{0} \hookrightarrow l_{1}(\Gamma)$. Thus, according to [18, Theorem 2.3], there exists $1 \leq m<\omega$ such that $l_{1}(\Gamma) \stackrel{c}{\hookrightarrow} Y^{m}$. Consequently $l_{\infty}(\Gamma) \hookrightarrow\left(Y^{*}\right)^{m}$, and applying Lemma 4.1 , we conclude $l_{\infty}(\Gamma) \hookrightarrow Y^{*}$. Hence by [34, Corollary 1.2], $l_{1}(\Gamma) \stackrel{c}{\hookrightarrow} Y$, a contradiction to the hypothesis of the lemma.

Lemma 4.4. Suppose that $X$ is an $\mathcal{N}_{\infty}$-space and that $Y$ is a Banach space containing no subspace isomorphic to $c_{0}$. Assume that $X^{*}$ or $Y$ has the approximation property and

$$
l_{1}\left([0, \lambda], X^{*}\right) \hat{\otimes}_{\pi} Y^{\xi} \sim l_{1}\left([0, \mu], X^{*}\right) \hat{\otimes}_{\pi} Y^{\eta},
$$

for some infinite ordinals $\lambda, \mu, \xi$ and $\eta$. Then $\bar{\xi}=\bar{\eta}$.

Proof. Suppose $\bar{\xi} \neq \bar{\eta}$ and assume without loss of generality that $\bar{\xi}<\bar{\eta}$. First of all, observe that $\mathbb{R}$ is a complemented subspace of $X^{*}$. Then by [14, page 41] and [38, section 20.3.7.B], $Y^{\eta} \sim \mathbb{R} \hat{\otimes}_{\pi} Y^{\eta} \stackrel{c}{\hookrightarrow} X^{*} \hat{\otimes}_{\pi} Y^{\eta}$. Hence by using our hypothesis we see that

$$
\mathbb{R}^{\eta} \hookrightarrow Y^{\eta} \stackrel{c}{\hookrightarrow} X^{*} \hat{\otimes}_{\pi} Y^{\eta} \stackrel{c}{\hookrightarrow} l_{1}\left([0, \mu], X^{*} \hat{\otimes}_{\pi} Y^{\eta}\right) \sim l_{1}\left([0, \lambda], X^{*} \hat{\otimes}_{\pi} Y^{\xi}\right) .
$$


Since $l_{1} \hookrightarrow \mathbb{R}^{\eta}$, there exists $1 \leq m<\omega$ such that $\mathbb{R}^{\eta} \hookrightarrow\left(X^{*} \hat{\otimes}_{\pi} Y^{\xi}\right)^{m}$. But by [38, section 20.3.3], we deduce

$$
\left(X^{*} \hat{\otimes}_{\pi} Y^{\xi}\right)^{m} \sim X^{*} \hat{\otimes}_{\pi}\left(Y^{\xi}\right)^{m} \sim X^{*} \hat{\otimes}_{\pi} Y^{\xi m} .
$$

Consequently, $\mathbb{R}^{\eta} \hookrightarrow X^{*} \hat{\otimes}_{\pi} Y^{\xi m}$. That is, $\mathbb{R}^{\eta} \hookrightarrow \mathcal{N}\left(X, Y^{\xi m}\right)$. Since $X$ is an $\mathcal{N}_{\infty}-$ space, there exists $1 \leq p<\omega$ such that

$$
\mathbb{R}^{\eta} \hookrightarrow\left(Y^{\xi m}\right)^{p} \sim Y^{\xi(m p)} \sim\left(Y^{m p}\right)^{\xi} .
$$

Now let $\alpha$ be the initial ordinal of cardinality $\bar{\eta}$. Thus by (24) we have $\mathbb{R}^{\alpha} \hookrightarrow$ $\left(Y^{m p}\right)^{\xi}$. Thus by [19, Proposition 2.13], we see that $c_{0} \hookrightarrow Y^{m p}$. Consequently by [35, Theorem 1], $c_{0} \hookrightarrow Y$. This contradiction completes the proof.

Before presenting the proof of Theorem 1.10, we recall some definitions from 17 and 20]. Let $\gamma$ be an ordinal. A $\gamma$-sequence in a set $A$ is a function $f:[1, \gamma[\rightarrow A$ and will be denoted by $\left(x_{\theta}\right)_{\theta<\gamma}$. If $A$ is a topological space and $\beta$ is an ordinal, we will say that the $\gamma$-sequence $\left(x_{\theta}\right)_{\theta<\gamma}$ is $\beta$-continuous if for every $\beta$-sequence of ordinals $\left(\theta_{\xi}\right)_{\xi<\beta}$ of $[0, \gamma]$ which converges to $\theta_{\beta}$ when $\xi$ converges to $\beta$, we have that $x_{\theta_{\xi}}$ converges to $x_{\theta_{\beta}}$.

Let $X$ be a Banach space, $\alpha$ an ordinal number and $\varphi$ a cardinal number. By $X_{\alpha}^{\varphi}$ we will denote the space of all $x^{* *} \in X^{* *}$ having the following property: for every set $B$ with $|B|=\varphi, \beta<\alpha$ and $B$-family $x^{b}=\left(x_{\xi}^{*}(b)\right)_{\xi<\beta}, b \in B$, of $\beta$-sequences of $X^{*}$ such that there exists $M \in \mathbb{R}$ with $\left\|x_{\xi}^{*}(b)\right\| \leq M$ for every $b \in B$ and $\xi<\beta$ and such that $x_{\xi}^{*}(b)(x) \stackrel{\xi \rightarrow \beta}{\longrightarrow} 0, \forall x \in X$, uniformly in $b$, we have $x^{* *}\left(x_{\xi}^{*}(b)\right) \stackrel{\xi \rightarrow \beta}{\longrightarrow} 0$ uniformly in $b$.

Clearly $X_{\alpha}^{\varphi}$ is a closed subspace of $X^{* *}$ and $c X \subset X_{\alpha}^{\varphi}$, where $c X$ is the canonical image of $X$ in $X^{* *}$.

Observe that if $X$ has the Mazur property, then $X_{\alpha}^{\varphi}=c X$.

Let $X$ be a Banach space and let $\alpha$ be an uncountable regular ordinal. Following [20, Definition 2.2], we set $[X]_{\alpha}=\bigcap_{\varphi<\alpha} X_{\alpha}^{\varphi}$.

Proof of Theorem 1.10. We begin by noticing that if $\lambda, \mu, \xi$ and $\eta$ are infinite ordinals, then by [11, Corollary 1, page 65],

$$
\mathcal{N}\left(X^{\lambda}, Y^{\xi}\right) \sim l_{1}\left([0, \lambda], X^{*}\right) \hat{\otimes}_{\pi} X^{\xi} \text { and } \mathcal{N}\left(X^{\mu}, Y^{\eta}\right) \sim l_{1}\left([0, \mu], X^{*}\right) \hat{\otimes}_{\pi} Y^{\eta} .
$$

First assume that Theorem 1.10(b) holds. So $\bar{\lambda}=\bar{\mu}$. We distinguish two cases:

Case 1. $\mathbb{R}^{\xi} \sim \mathbb{R}^{\eta}$. Then $Y^{\xi} \sim Y \hat{\otimes}_{\epsilon} \mathbb{R}^{\xi} \sim Y \hat{\otimes}_{\epsilon} \mathbb{R}^{\eta} \sim Y^{\eta}$. Hence

$$
l_{1}\left([0, \lambda], X^{*}\right) \hat{\otimes}_{\pi} Y^{\xi} \sim l_{1}\left([0, \mu], X^{*}\right) \hat{\otimes}_{\pi} Y^{\eta},
$$

and by (25) it follows that Theorem 1.10(a) holds.

Case 2. $\mathbb{R}^{\xi} \sim \mathbb{R}^{\alpha p}$ and $\mathbb{R}^{\eta} \sim \mathbb{R}^{\alpha q}$ for some uncountable regular ordinal $\alpha$ and finite ordinals $p$ and $q$. Then $Y^{\xi} \sim Y^{\alpha p}$ and $Y^{\eta} \sim Y^{\alpha q}$. Hence by [38, section 20.7.B],

and

$$
l_{1}\left([0, \lambda], X^{*}\right) \hat{\otimes}_{\pi} Y^{\xi} \sim l_{1}\left([0, \lambda], X^{*}\right) \hat{\otimes}_{\pi} Y^{\alpha p},
$$

Since $\lambda$ is infinite, it is easy to see that $l_{1}\left([0, \lambda], X^{*}\right) \sim\left(l_{1}\left([0, \lambda], X^{*}\right)\right)^{q}$. Therefore by [38, section 20.3.7.B],

$$
l_{1}\left([0, \lambda], X^{*}\right) \hat{\otimes}_{\pi} Y^{\alpha p} \sim\left(l_{1}\left([0, \lambda], X^{*}\right)\right)^{q} \hat{\otimes}_{\pi} Y^{\alpha p} .
$$


Now by applying [38, Section 20.3.3] twice, we get

$$
\left(l_{1}\left([0, \lambda], X^{*}\right)\right)^{q} \hat{\otimes}_{\pi} Y^{\alpha p} \sim\left(l_{1}\left([0, \lambda], X^{*}\right) \hat{\otimes}_{\pi} Y^{\alpha p}\right)^{q} \sim l_{1}\left([0, \lambda], X^{*}\right) \hat{\otimes}_{\pi}\left(Y^{\alpha p}\right)^{q} .
$$

According to $(28)$ and $(29)$

$$
l_{1}\left([0, \lambda], X^{*}\right) \hat{\otimes}_{\pi} Y^{\alpha p} \sim l_{1}\left([0, \lambda], X^{*}\right) \hat{\otimes}_{\pi}\left(Y^{\alpha p}\right)^{q} .
$$

Similarly, we obtain

$$
l_{1}\left([0, \mu], X^{*}\right) \hat{\otimes}_{\pi} Y^{\alpha q} \sim l_{1}\left([0, \mu], X^{*}\right) \hat{\otimes}_{\pi}\left(Y^{\alpha q}\right)^{p} .
$$

Moreover, since $\left(Y^{\alpha p}\right)^{q} \sim Y^{\alpha(p q)} \sim\left(Y^{\alpha q}\right)^{p}$, it follows [38, section 20.3.7B] that

$$
l_{1}\left([0, \lambda], X^{*}\right) \hat{\otimes}_{\pi}\left(Y^{\alpha p}\right)^{q} \sim l_{1}\left([0, \mu], X^{*}\right) \hat{\otimes}_{\pi}\left(Y^{\alpha p}\right)^{q} .
$$

Keeping in mind that $\bar{\lambda}=\bar{\mu}$, by (26), (27), (30), (31), (32) and [38, Section 20.3.7B], we get

$$
l_{1}\left([0, \lambda], X^{*}\right) \hat{\otimes}_{\pi} Y^{\xi} \sim l_{1}\left([0, \lambda], X^{*}\right) \hat{\otimes}_{\pi} Y^{\eta} \sim l_{1}\left([0, \mu], X^{*}\right) \hat{\otimes}_{\pi} Y^{\eta},
$$

and again by (25) we deduce that Theorem 1.10(a) holds.

Conversely, suppose now that (a) holds. Thus by (25),

$$
l_{1}\left([0, \lambda], X^{*}\right) \hat{\otimes}_{\pi} Y^{\xi} \sim l_{1}\left([0, \mu], X^{*}\right) \hat{\otimes}_{\pi} Y^{\eta} .
$$

Thus Lemma 4.3 implies that $\bar{\lambda}=\bar{\mu}$. Moreover, by Lemma $4.4, \bar{\xi}=\bar{\eta}$.

Next, without loss of generality we assume that $\xi \leq \eta$. Let $\alpha$ be the initial ordinal of cardinality $\bar{\xi}$. There are two cases:

Case 1. $\alpha=\omega$ or $\alpha$ is a singular ordinal or $\alpha$ is an uncountable regular ordinal with $\alpha^{2} \leq \xi$. In this case $\eta<\xi^{\omega}$ because if $\xi^{\omega} \leq \eta$, then by proceeding as in (24), there exist finite ordinals $m$ and $p$ such that $\mathbb{R}^{\xi^{\omega}} \hookrightarrow \mathbb{R}^{\eta} \hookrightarrow\left(Y^{m p}\right)^{\xi}$. Thus by [19, Lemma 2.11], we conclude that $c_{0} \hookrightarrow Y^{m p}$. Once again by 35, Theorem 1], we infer that $c_{0} \hookrightarrow Y$, which is absurd. Thus $\eta<\xi^{\omega}$ and [28, Theorem 1] implies that $\mathbb{R}^{\xi} \sim \mathbb{R}^{\eta}$. So Theorem 1.10(b) holds.

Case 2. $\alpha$ is an uncountable regular ordinal with $\xi<\alpha^{2}$. In this case we will show that $\eta<\alpha^{2}$. Indeed, otherwise write $\xi=\alpha \xi^{\prime}+\gamma$ with $\xi^{\prime}<\alpha$. Then [28, Theorem 2] implies that $Y^{\xi} \sim Y^{\alpha \xi^{\prime}}$. So by (33)

$$
\mathbb{R}^{\alpha^{2}} \hookrightarrow \mathbb{R}^{\eta} \hookrightarrow Y^{\eta} \stackrel{c}{\hookrightarrow} X^{*} \hat{\otimes}_{\pi} Y^{\eta} \stackrel{c}{\hookrightarrow} l_{1}\left([0, \mu], X^{*}\right) \hat{\otimes}_{\pi} Y^{\eta} \sim l_{1}\left([0, \lambda], X^{*}\right) \hat{\otimes}_{\pi} Y^{\alpha \xi^{\prime}} .
$$

Once again proceeding as in (24) we conclude that there exists finite ordinals $m$ and $p$ such that $\mathbb{R}^{\alpha^{2}} \hookrightarrow\left(Y^{m p}\right)^{\alpha \xi^{\prime}}$.

Let $I$ and $J$ be two sets of cardinality $\bar{\alpha}$ and $\bar{\xi}^{\prime}$ respectively. By [19, Lemma 2.4] we see that

$$
\frac{\left[\mathbb{R}^{\alpha^{2}}\right]_{\alpha}}{c\left(\mathbb{R}^{\alpha^{2}}\right)} \hookrightarrow \frac{\left[\left(Y^{m p}\right)^{\alpha \xi^{\prime}}\right]_{\alpha}}{c\left(\left(Y^{m p}\right)^{\alpha \xi^{\prime}}\right)}
$$

Now by [20, Proposition 2.8],

$$
c_{0}(I) \sim \frac{\left[\mathbb{R}^{\alpha^{2}}\right]_{\alpha}}{c\left(\mathbb{R}^{\alpha^{2}}\right)} \text { and } c_{0}\left(J, Y^{m p}\right) \sim \frac{\left[\left(Y^{m p}\right)^{\alpha \xi^{\prime}}\right]_{\alpha}}{c\left(\left(Y^{m p}\right)^{\alpha \xi^{\prime}}\right)} .
$$

Consequently, $c_{0}(I) \hookrightarrow c_{0}\left(J, Y^{m p}\right)$. According to [19, Lemma 2.4], we conclude that $c_{0} \hookrightarrow Y^{m p}$ and therefore [35, Theorem 1] implies that $c_{0} \hookrightarrow Y$, contradicting the hypothesis. 
So $\eta<\alpha^{2}$. Write also $\eta=\alpha \eta^{\prime}+\delta$ with $\eta^{\prime}, \delta<\alpha$. According to [28, Theorem 2] $Y^{\xi} \sim Y^{\alpha \xi^{\prime}}$ and $Y^{\eta} \sim Y^{\alpha \eta^{\prime}}$. So by [38, section 20.3.7B],

$$
l_{1}\left([0, \lambda], X^{*}\right) \hat{\otimes}_{\pi} Y^{\xi} \sim l_{1}\left([0, \lambda], X^{*}\right) \hat{\otimes}_{\pi} Y^{\alpha \xi^{\prime}},
$$

and

$$
l_{1}\left([0, \mu], X^{*}\right) \hat{\otimes}_{\pi} Y^{\eta} \sim l_{1}\left([0, \mu], X^{*}\right) \hat{\otimes}_{\pi} Y^{\alpha \eta^{\prime}} .
$$

On the other hand, by [38, section 20.3.3] and [14, Theorem 1.10] we know that

$$
l_{1}\left([0, \mu], X^{*}\right) \hat{\otimes}_{\pi} Y^{\alpha \eta^{\prime}} \sim\left(l_{1}[0, \mu] \hat{\otimes}_{\pi} X^{*}\right) \hat{\otimes}_{\pi} Y^{\alpha \eta^{\prime}} \sim l_{1}\left([0, \mu], X^{*} \hat{\otimes}_{\pi} Y^{\alpha \eta^{\prime}}\right) .
$$

Hence

$$
\mathbb{R}^{\alpha \xi^{\prime}} \hookrightarrow Y^{\alpha \xi^{\prime}} \stackrel{c}{\hookrightarrow} X^{*} \hat{\otimes}_{\pi} Y^{\alpha \xi^{\prime}} \stackrel{c}{\hookrightarrow} l_{1}\left([0, \lambda], X^{*} \hat{\otimes}_{\pi} Y^{\alpha \xi^{\prime}}\right) \sim l_{1}\left([0, \mu], X^{*}\right) \hat{\otimes}_{\pi} Y^{\alpha \eta^{\prime}} .
$$

Proceeding as in (24) we infer that there exist finite ordinals $m$ and $p$ such that $\mathbb{R}^{\alpha \xi^{\prime}} \hookrightarrow\left(Y^{m p}\right)^{\alpha \eta^{\prime}}$.

Let $I$ and $J$ be two sets of cardinality $\bar{\xi}^{\prime}$ and $\bar{\eta}^{\prime}$ respectively. According to [20, Lemma 2.4] we have

$$
\frac{\left[\mathbb{R}^{\alpha \xi^{\prime}}\right]_{\alpha}}{c\left(\mathbb{R}^{\alpha \xi^{\prime}}\right)} \hookrightarrow \frac{\left[\left(Y^{m p}\right)^{\alpha \eta^{\prime}}\right]_{\alpha}}{c\left(\left(Y^{m p}\right)^{\alpha \eta^{\prime}}\right)}
$$

But by [20, Proposition 2.8],

$$
c_{0}(I) \sim \frac{\left[\mathbb{R}^{\alpha \xi^{\prime}}\right]_{\alpha}}{c\left(\mathbb{R}^{\alpha \xi^{\prime}}\right)} \text { and } c_{0}\left(J, Y^{m p}\right) \sim \frac{\left[\left(Y^{m p}\right)^{\alpha \eta^{\prime}}\right]_{\alpha}}{c\left(\left(Y^{m p}\right)^{\alpha \eta^{\prime}}\right)} .
$$

Hence $c_{0}(I) \hookrightarrow c_{0}\left(J, Y^{m p}\right)$. Since $Y$ contains no subspace isomorphic to $c_{0}$, it follows that the same happens with $Y^{m p}$. Therefore by [19, Lemma 2.4], we conclude that $\xi^{\prime}$ is finite if and only if $\eta^{\prime}$ is finite. In this case, $\mathbb{R}^{\xi} \sim \mathbb{R}^{\alpha \xi^{\prime}}$ and $\mathbb{R}^{\eta} \sim \mathbb{R}^{\alpha \eta^{\prime}}$. Consequently (b) holds. Otherwise, if $\xi^{\prime}$ is infinite, then again by [19, Lemma 2.4], $\bar{\xi}^{\prime}=\bar{\eta}^{\prime}$. Hence by [28, Theorem 2], $\mathbb{R}^{\xi} \sim \mathbb{R}^{\eta}$ and again Theorem 1.10(b) holds. Thus the theorem is proved.

\section{On ISOMORPhic Classifications of $\mathcal{K}\left(X^{\lambda}, Y^{\xi}\right)$ SPACES}

The purpose of this section is to prove the generalization of (4).

Lemma 5.1. Let $X$ be a Banach space such that $X^{*}$ is isomorphic to a subspace of $l_{1}$ and let $Y$ be a Banach space containing no subspace isomorphic to $c_{0}$. Then for every set $\Gamma$ we have $c_{0} \nrightarrow l_{1}\left(\Gamma, X^{*}\right) \hat{\otimes}_{\epsilon} Y$.

Proof. Suppose that there exists a set $\Gamma$ such that $c_{0} \hookrightarrow l_{1}\left(\Gamma, X^{*}\right) \hat{\otimes}_{\epsilon} Y$. Since $X^{*} \hookrightarrow l_{1}$, we infer $c_{0} \hookrightarrow l_{1}\left(\Gamma, X^{*}\right) \hat{\otimes}_{\epsilon} Y \hookrightarrow l_{1}\left(\Gamma, l_{1}\right) \hat{\otimes}_{\epsilon} Y \sim l_{1}(\Gamma) \hat{\otimes}_{\epsilon} Y$. Then by the separability of $c_{0}$, we deduce $c_{0} \hookrightarrow l_{1} \hat{\otimes}_{\epsilon} Y \hookrightarrow L_{1}[0,1] \hat{\otimes}_{\epsilon} Y$. Hence by [16, Theorem 3.2] we would conclude that $c_{0} \hookrightarrow Y$, which contradicts our hypothesis regarding $Y$ and this finishes the proof.

Lemma 5.2. Let $X$ be a Banach space such that $X^{*}$ is isomorphic to a subspace of $l_{1}$ and let $Y$ be a $\mathcal{K}_{0}$-space containing no subspace isomorphic to $c_{0}$. Suppose that

$$
l_{1}\left([0, \lambda], X^{*}\right) \hat{\otimes}_{\epsilon} Y^{\xi} \sim l_{1}\left([0, \mu], X^{*}\right) \hat{\otimes}_{\epsilon} Y^{\eta},
$$

for some infinite ordinals $\lambda, \mu, \xi$ and $\eta$. Then $\bar{\lambda}=\bar{\mu}$. 
Proof. It follows from the hypothesis that

$$
l_{1}\left([0, \lambda], X^{*}\right) \hat{\otimes}_{\epsilon} Y \stackrel{c}{\hookrightarrow} l_{1}\left([0, \mu], X^{*}\right) \hat{\otimes}_{\epsilon} Y^{\eta} \sim\left(l_{1}\left([0, \mu], X^{*}\right) \hat{\otimes}_{\epsilon} Y\right)^{\eta} .
$$

Since $l_{1}\left([0, \mu], X^{*}\right) \hat{\otimes}_{\epsilon} Y$ is isomorphic to its square, it follows by (34), Lemma 5.1 and [18, Theorem 2.3] that $l_{1}\left([0, \lambda], X^{*}\right) \hat{\otimes}_{\epsilon} Y \stackrel{c}{\hookrightarrow} l_{1}\left([0, \mu], X^{*}\right) \hat{\otimes}_{\epsilon} Y$.

Analogously we obtain $l_{1}\left([0, \mu], X^{*}\right) \hat{\otimes}_{\epsilon} Y \stackrel{c}{\hookrightarrow} l_{1}\left([0, \lambda], X^{*}\right) \hat{\otimes}_{\epsilon} Y$. Therefore by the Pełczyński decomposition method [9, page 63], we infer

$$
l_{1}\left([0, \mu], X^{*}\right) \hat{\otimes}_{\epsilon} Y \sim l_{1}\left([0, \lambda], X^{*}\right) \hat{\otimes}_{\epsilon} Y .
$$

By hypothesis $X^{*} \hookrightarrow l_{1}$. So (35) implies that

$$
l_{1}[0, \lambda] \hat{\otimes}_{\epsilon} Y \hookrightarrow l_{1}[0, \mu] \hat{\otimes}_{\epsilon} Y \text { and } l_{1}[0, \mu] \hat{\otimes}_{\epsilon} Y \hookrightarrow l_{1}[0, \lambda] \hat{\otimes}_{\epsilon} Y .
$$

By the definition of $\mathcal{K}_{0}$-space we conclude that $\bar{\lambda}=\bar{\mu}$.

Proof of Theorem 1.13. We start by observing that [11, Proposition 5.3] implies that

$$
\mathcal{K}\left(X^{\lambda}, Y^{\xi}\right) \sim l_{1}\left([0, \lambda], X^{*}\right) \hat{\otimes}_{\epsilon} Y^{\xi} \text { and } \mathcal{K}\left(X^{\mu}, Y^{\eta}\right) \sim l_{1}\left([0, \mu], X^{*}\right) \hat{\otimes}_{\epsilon} Y^{\eta} .
$$

First suppose that Theorem 1.13(b) holds. Then it suffices to proceed as in the proof of the first part of Theorem 1.10 and to use the analogous properties of injective tensor product to conclude that Theorem 1.13(a) holds.

Conversely, assume that Theorem 1.13(a) holds. So by (36) we have

$$
l_{1}\left([0, \lambda], X^{*}\right) \hat{\otimes}_{\epsilon} Y^{\xi} \sim l_{1}\left([0, \mu], X^{*}\right) \hat{\otimes}_{\epsilon} Y^{\eta} .
$$

Then according to Lemma $4.3, \bar{\lambda}=\bar{\mu}$, and therefore by (37) we get

$$
l_{1}\left([0, \lambda], X^{*}\right) \hat{\otimes}_{\epsilon} Y^{\xi} \sim l_{1}\left([0, \lambda], X^{*}\right) \hat{\otimes}_{\epsilon} Y^{\eta} .
$$

Put $Z=l_{1}\left([0, \lambda], X^{*}\right) \hat{\otimes}_{\epsilon} Y$. Since $X^{*}$ is separable, by [27, Corollary 5.2.3], $X^{*} \hat{\otimes}_{\epsilon} Y$ has the Mazur property. Therefore [27, Theorem 5.3] implies that $Z$ also has this property. Notice that for every ordinal $\beta, l_{1}\left([0, \lambda], X^{*}\right) \hat{\otimes}_{\epsilon} Y^{\beta} \sim Z^{\beta}$. Hence (38) can be rewritten as

$$
Z^{\xi} \sim Z^{\eta}
$$

Moreover, by Lemma 5.1, $c_{0} \triangleleft Z$. Thus, we may apply [20, Theorem 1.1] in (39) to obtain that $\bar{\xi}=\bar{\eta}$.

Next, without loss of generality we suppose that $\xi \leq \eta$. Let $\alpha$ be the initial ordinal of cardinality $\bar{\xi}$. We distinguish two cases:

Case 1. $\alpha=\omega$ or $\alpha$ is a singular ordinal or $\alpha$ is an uncountable regular ordinal with $\alpha^{2} \leq \xi$. Then by (39) and [20, Theorem 1.1] we see that $\eta<\xi^{\omega}$. Further, in view of [28, Theorem 1] this is equivalent to $\mathbb{R}^{\xi} \sim \mathbb{R}^{\eta}$. So Theorem 1.13(b) holds.

Case 2. $\alpha$ is an uncountable regular ordinal with $\xi<\alpha^{2}$. In this case, in virtue of (39), [20, Theorem 1.1] implies that $\eta<\alpha^{2}$. Let $\xi^{\prime}, \eta^{\prime}, \gamma$ and $\delta$ be ordinals such that $\xi=\alpha \xi^{\prime}+\gamma$ and $\eta=\alpha \eta^{\prime}+\delta, \xi^{\prime}, \eta^{\prime} \leq \alpha$ and $\gamma, \delta<\alpha$. Then by (39) and 20, Theorem 1.1] we deduce that $\bar{\xi}^{\prime}$ and $\bar{\eta}^{\prime}$ are finite or $\bar{\xi}^{\prime}$ and $\bar{\eta}^{\prime}$ are infinite and they have the same cardinality.

By [28, Theorem 1] this is equivalent to $\mathbb{R}^{\xi} \sim \mathbb{R}^{\eta}$ or $\xi^{\prime}$ and $\eta^{\prime}$ are finite with $\xi^{\prime} \neq \eta^{\prime}$ and $\mathbb{R}^{\xi} \sim \mathbb{R}^{\alpha \xi^{\prime}}$ and $\mathbb{R}^{\eta} \sim \mathbb{R}^{\alpha \eta^{\prime}}$. So once again Theorem 1.13(b) holds. This completes the proof of the theorem. 


\section{ACKNOWLEDGMENT}

The authors would like to thank the referee for a careful reading of the paper and for helpful suggestions.

\section{REFERENCES}

1. D. E. Alspach, A quotient of $C\left(\omega^{\omega}\right)$ which is not isomorphic to a subspace of $C(\alpha), \alpha<\omega_{1}$. Israel J. Math. 35 (1980), 1-2, 49-60. MR576461 (81g:54012)

2. D. E. Alspach, Y. Benyamini, A geometrical property of $C(K)$ spaces. Israel J. Math. 64 (1988), 179-194. MR986146 (91b:46023)

3. S. A. Argyros and R. G. Haydon. A hereditarily indecomposable $\mathcal{L}_{\infty}$-space that solves the Scalar-Plus-Compact Problem. Acta Math. 206, 1 (2011), 1-54. MR 2784662

4. Y. Benyamini, J. Lindenstrauss, A predual of $l_{1}$ which is not isomorphic to a $C(K)$ space. Israel J. Math. 13 (1972), 246-259. MR0331013(48:9348)

5. C. Bessaga, A. Pełczyński, Spaces of continuous functions IV. Studia Math. XIX (1960), 53-61. MR0113132 (22:3971)

6. J. Bourgain, F. Delbaen, A class of special $\mathcal{L}^{\infty}$-spaces. Acta Math. 145 (1960), 155-176. MR $590288(82 \mathrm{~h}: 46023)$

7. P. Cembranos, J. Mendoza, $l_{\infty}\left(l_{1}\right)$ and $l_{1}\left(l_{\infty}\right)$ are not isomorphic. J. Math. Anal. Appl. 341 (2008), 1, 295-297. MR2394084 (2009a:46012)

8. P. Cembranos, J. Mendoza, On the mutually nonisomorphic $l_{p}\left(l_{q}\right)$ spaces. Math. Nachr., to appear.

9. P. G. Casazza, The Schroeder-Bernstein property for Banach spaces. Banach space theory (Iowa City, IA, 1987), 61-77, Contemp. Math., 85, Amer. Math. Soc., Providence, RI, 1989. MR.983381 (90d:46019)

10. P.G. Casazza, Approximation properties. Handbook of the geometry of Banach spaces I. North-Holland Publishing Co., Amsterdam, 2001, 271-316. MR.1863695 (2003f:46012)

11. A. Defant and K. Floret, Tensor norms and operator ideals. Math. Studies, 176, NorthHolland, Amsterdam, 1993. MR1209438 (94e:46130)

12. J. Diestel, J.J.J.R. Uhl, Vector Measures. Mathematical Surveys, 15, Amer. Math. Soc., Providence, RI, 1977. MR0453964 (56:12216)

13. J. Diestel, H. Jarchow, A. Tonge, Absolutely summing operators. Cambridge Studies in Advanced Mathematics, 43, Cambridge University Press, Cambridge, 1995. MR.1342297 (96i:46001)

14. J. Diestel, J. Fourier, J. Swart. The Projective Tensor Product I. Contemporary Math., 321, Amer. Math. Soc., Providence, RI, 2003, 37-65. MR1978806 (2005a:46040)

15. G. A. Edgar, Measurability in a Banach space II. Indiana Univ. Math. 28 (1977), 559-579. MR542944 (81d:28016)

16. F. J. Freniche, Embedding $c_{0}$ in the space of Pettis integrable functions. Quaest. Math. 21 (1998), 3-4, 261-267. MR 1701785 (2000d:46047)

17. E. M. Galego, How to generate new Banach spaces non-isomorphic to their Cartesian squares. Bull. Polish Acad. Sci. Math. 47 (1999), 1, 21-25. MR1685684 (2001b:46015)

18. E. M. Galego, On subspaces and quotients of Banach spaces $C(K, X)$. Monatsh. Math. 136 (2002), 2, 87-97. MR1914222 (2003g:46038)

19. E. M. Galego, On isomorphic classifications of compact operators. Proc. Amer. Math. Soc. 137 (2009), 3335-3342. MR 2515403 (2010d:46010)

20. E. M. Galego, Complete isomorphic classification of some spaces of compact operators. Proc. Amer. Math. Soc. 138 (2010), 725-736. MR2557189 (2010j:46018)

21. R. J. Gardner, W. F. Pfeffer. Borel measures. Handbook of set-theoretic topology. NorthHolland, Amsterdam, 1984, 961-1043. MR776641 (86c:28031)

22. S. P. Gul'ko, A. V. Os'kin, Isomorphic classification of spaces of continuous functions on totally ordered bicompacta. Functional Anal. Appl. 9 (1975), 1, 56-57. MR0377489 (51:13661)

23. S. Heinrich, Approximation properties in tensor products. Mat. Zametki 17 (1975), 459-466. MR0412835 (54:956)

24. T. Jech, Set Theory. Pure and Applied Mathematics. Academic Press [Harcourt Brace Jovanovich, Publishers], New York-London, 1978. MR.506523(80a:03062) 
25. W. B. Johnson, J. Lindenstrauss, Handbook of the geometry of Banach spaces. North-Holland Publishing Co., Amsterdam, 2001, 1-84. MR.1863689 (2003f:46013)

26. A. Kanamori, M. Magidor, The evolution of large cardinal axioms in set theory. Higher set theory (Proc. Conf., Math. Forschungsinst., Oberwolfach, 1977), Lecture Notes in Math., 669, Springer, Berlin, 1978, 99-275. MR.520190 (80b:03083)

27. T. Kappeler, Banach spaces with the condition of Mazur. Math. Z. 191 (1986), 623-631. MR $832820(87 \mathrm{~h}: 46040)$

28. S. V. Kislyakov, Classification of spaces of continuous functions of ordinals. Siberian Math. J. 16 (1975), 2, 226-231.

29. M. A. Labbé, Isomorphism of continuous functions. Studia Math. LII (1975), 221-231. MR0390737 (52:11560)

30. J. Lindenstrauss, L. Tzafriri, Classical Banach spaces I. Sequence Spaces. Springer-Verlag, Berlin-New York, 1977. MR0500056 (58:17766)

31. D. Leung, Banach spaces with Mazur's property. Glasgow Math. J. 33 (1991), 51-54. MR.1089953 (92b:46021)

32. S. Mazurkiewicz, W. Sierpiński. Contribution à la topologie des ensembles dénombrables. Fund. Math. 1 (1920), 17-27.

33. H. P. Rosenthal, On relatively disjoint families of measures, with some applications to Banach space theory. Studia Math. 37 (1970), 13-36. MR0270122 (42:5015)

34. H. P. Rosenthal, On injective Banach spaces and the spaces $L^{\infty}(\mu)$ for finite measure $\mu$. Acta Math. 124 (1970), 205-248. MR0257721 (41:2370)

35. C. Samuel, Sur la reproductibilité des espaces $l_{p}$. Math. Scand. 45 (1979), 103-117. MR567436 (81e:46062)

36. C. Samuel, On spaces of operators on $C(Q)$ spaces ( $Q$ countable metric space). Proc. Amer. Math. Soc. 137 (2009), 3, 965-970. MR2457436 (2009m:46004)

37. Z. Semadeni, Banach spaces non-isomorphic to their Cartesian squares II. Bull. Acad. Polon. Sci. Ser. Sci. Math. Astr. Phys. 8 (1960), 81-84. MR0115074 (22:5877)

38. Z. Semadeni, Banach spaces of continuous functions. Vol. I. Monografie Matematyczne, Tom 55, PWN-Polish Scientific Publishers, Warsaw, 1971. MR0296671 (45:5730)

39. A. Wilansky, Mazur spaces. Internat. J. Math. Math. Sci. 4 (1981), 39-53. MR606656 (82f:46001)

Department of Mathematics, University of São Paulo, São Paulo, Brazil 05508-090

E-mail address: eloi@ime.usp.br

Department of Mathematics, University of SÃo Paulo, São Paulo, Brazil 05508-090

E-mail address: ronaldep@ime.usp.br 